Author affiliations appear at the end of this article.

Published online ahead of print at www.jco.org on March 17, 2014.

Supported by Fonds Cancer (Belgium); Dutch Cancer Society (the Netherlands); Institut National du Cancer Fondation Contre le Cancer, Assistance Publique Hopitaux Paris, and Societe Française de Medecine Nucleaire et Imagerie Moleculaire (France); Associazone Angela Serra (Italy); and Chugai Pharmaceutical (Japan).

M.M. and C.F. contributed equally to this work.

tDeceased

Presented (preliminary results) at the 51 st Annual Meeting of the American Society of Hematology (ASH), New Orleans, LA, December 5-8, 2009; Eighth International Symposium on Hodgkin Lymphoma, Cologne, Germany, October 23-26, 2010; and 54th ASH Annual Meeting, Atlanta, GA December 8-11, 2012.

Authors' disclosures of potential conflicts of interest and author contributions are found at the end of this article.

Clinical trial information: NCT00433433.

Corresponding author: John M.M. Raemaekers, MD, PhD, Department of Hematology, Radboud University Medical Center, PO Box 9101, 6500HB Nijmegen, the Netherlands; e-mail: john.raemaekers@radboudumc.nl.

(C) 2014 by American Society of Clinical Oncology

0732-183X/14/3212w-1188w/\$20.00

DOI: 10.1200/JCO.2013.51.9298

\title{
Omitting Radiotherapy in Early Positron Emission Tomography-Negative Stage I/II Hodgkin Lymphoma Is Associated With an Increased Risk of Early Relapse: Clinical Results of the Preplanned Interim Analysis of the Randomized EORTC/LYSA/FIL H10 Trial
}

John M.M. Raemaekers, Marc P.E. André, Massimo Federico, Theodore Girinsky, Reman Oumedaly, Ercole Brusamolino, † Pauline Brice, Christophe Fermé, Richard van der Maazen, Manuel Gotti, Reda Bouabdallah, Catherine J. Sebban, Yolande Lievens, Allessandro Re, Aspasia Stamatoullas, Frank Morschhauser, Pieternella J. Lugtenburg, Elisabetta Abruzzese, Pierre Olivier, Rene-Olivier Casasnovas, Gustaaf van Imhoff, Tiana Raveloarivahy, Monica Bellei, Thierry van der Borght, Stephane Bardet, Annibale Versari, Martin Hutchings, Michel Meignan, and Catherine Fortpied

Processed as a Rapid Communication manuscript. See accompanying editorial on page 1180

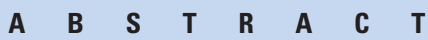

\section{Purpose}

Combined-modality treatment is standard treatment for patients with clinical stage I/II Hodgkin lymphoma $(\mathrm{HL})$. We hypothesized that an early positron emission tomography (PET) scan could be used to adapt treatment. Therefore, we started the randomized EORTC/LYSA/FIL Intergroup $\mathrm{H} 10$ trial evaluating whether involved-node radiotherapy (IN-RT) could be omitted without compromising progression-free survival in patients attaining a negative early PET scan after two cycles of ABVD (doxorubicin, bleomycin, vinblastine, and dacarbazine) as compared with standard combined-modality treatment.

\section{Patients and Methods}

Patients age 15 to 70 years with untreated clinical stage I/II HL were eligible. Here we report the clinical outcome of the preplanned interim futility analysis scheduled to occur after documentation of 34 events in the early PET-negative group. Because testing for futility in this noninferiority trial corresponds to testing the hypothesis of no difference, a one-sided superiority test was conducted.

\section{Results}

The analysis included 1,137 patients. In the favorable subgroup, 85.8\% had a negative early PET scan (standard arm, one event $v$ experimental arm, nine events). In the unfavorable subgroup, $74.8 \%$ had a negative early PET scan (standard arm, seven events $v$ experimental arm, 16 events). The independent data monitoring committee concluded it was unlikely that we would show noninferiority in the final results for the experimental arm and advised stopping random assignment for early PET-negative patients

\section{Conclusion}

On the basis of this analysis, combined-modality treatment resulted in fewer early progressions in clinical stage $\mathrm{I} / \mathrm{II} \mathrm{HL}$, although early outcome was excellent in both arms. The final analysis will reveal whether this finding is maintained over time.

\section{J Clin Oncol 32:1188-1194. (C) 2014 by American Society of Clinical Oncology}

\section{INTRODUCTION}

Standard treatment for patients with clinical stage I/II Hodgkin lymphoma (HL) consists of the combination of chemotherapy (mostly ABVD [doxorubicin, bleomycin, vinblastine, and dacarbazine]) followed by radiotherapy (RT). ${ }^{1,2}$ Despite the reduc- tion of RT fields from extended field to involved node (IN), ${ }^{3}$ there has been a continuing attempt to omit RT to reduce late complications. ${ }^{4}$ Prognostic factors have yet to reproducibly identify those who can be treated with chemotherapy alone. Patients have a significantly higher risk of recurrence after chemotherapy alone than after combined-modality 
treatment. ${ }^{5-7}$ Because of adequate but intensive salvage treatment, overall survival does not differ significantly. The right balance between initial cure through combined-modality treatment-accepting a higher risk of late complications_-and a higher recurrence rate after omitting RT in subsets of patients who will subsequently need intensive salvage treatment (often including autologous stemcell transplantation) is a matter of an unsettled debate. ${ }^{8-12}$ Early $\left[{ }^{18} \mathrm{~F}\right]$ fluorodeoxyglucose-positron emission tomography (PET) scanning after two cycles of chemotherapy provides prognostic information on final outcome; patients who attain a negative early PET scan after two cycles of ABVD (or similar) chemotherapy have better progression-free survival (PFS) than those with a positive early PET scan, when the initially planned treatment is completed. ${ }^{13-15}$ Patients with a negative early PET scan might be treated less intensively in view of the excellent prognosis. We hypothesized that patients with stage I/II disease who achieve a negative early PET scan could be spared additional RT. In contrast, those with a positive early PET scan might benefit from early treatment intensification ${ }^{16}$ from ABVD to BEACOPP (bleomycin, etoposide, doxorubicin, cyclophosphamide, vincristine, procarbazine, and prednisone) escalated.

We initiated a randomized controlled phase III intergroup trial to evaluate primarily the efficacy of chemotherapy alone versus combined-modality treatment in patients with a negative early PET scan after two cycles of ABVD. Second, we evaluated the efficacy of intensification of chemotherapy in patients with a positive early PET scan after two cycles of ABVD. ${ }^{17}$ A preplanned interim analysis was conducted after a predefined number of events had occurred during the course of the trial to detect possible worse early outcome of the experimental arm. Our report describes the results of this preplanned interim futility analysis performed among patients with a negative early PET scan.

\section{PATIENTS AND METHODS}

\section{Study Design}

In October 2006, the European Organisation for Research and Treatment of Cancer (EORTC) Lymphoma Group and the Lymphoma Study Association (LYSA), formerly GELA (Groupe d'Étude de Lymphomes Adultes), launched the H10 randomized intergroup trial (EORTC 20051), which the Intergruppo Italiano Linfomi, now called Fondazione Italiana Linfomi (FIL), joined in March 2008. The main objective was to evaluate whether IN-RT could be omitted without loss of efficacy in terms of PFS in patients with stage I/II HL who attain a negative early PET scan after two cycles of ABVD (Fig 1). The secondary objective was to evaluate whether intensification from ABVD to BEACOPP escalated would increase PFS in patients who did not achieve a negative early PET scan. Eligibility criteria included previously untreated patients with histologically proven classical HL, supradiaphragmatic Ann Arbor stage I/II disease, age 15 to 70 years, WHO performance status of 0 to 3, and written informed consent. The number of chemotherapy cycles depended on the prognostic subgroup: favorable or unfavorable (Fig 1). ${ }^{18,19}$

The study was approved by the respective scientific boards and national ethics committees and was registered in ClinicalTrials.gov. ${ }^{20}$ Patients were centrally randomly assigned to receive either standard or investigational treatment. A minimization technique was used for treatment allocation in each prognostic subgroup (favorable $v$ unfavorable), stratifying by institution, Ann Arbor stage (I $v$ II), and availability of baseline PET.

\section{Early PET Scan}

A prospective central reading of the early PET scan was planned in the protocol. $^{21}$ For technical reasons, centralized review for the LYSA group started from the initiation of the trial, and for EORTC/FIL groups, it occurred from 2008 onward. In case of absence of a timely ( $<72$ hours) centralized

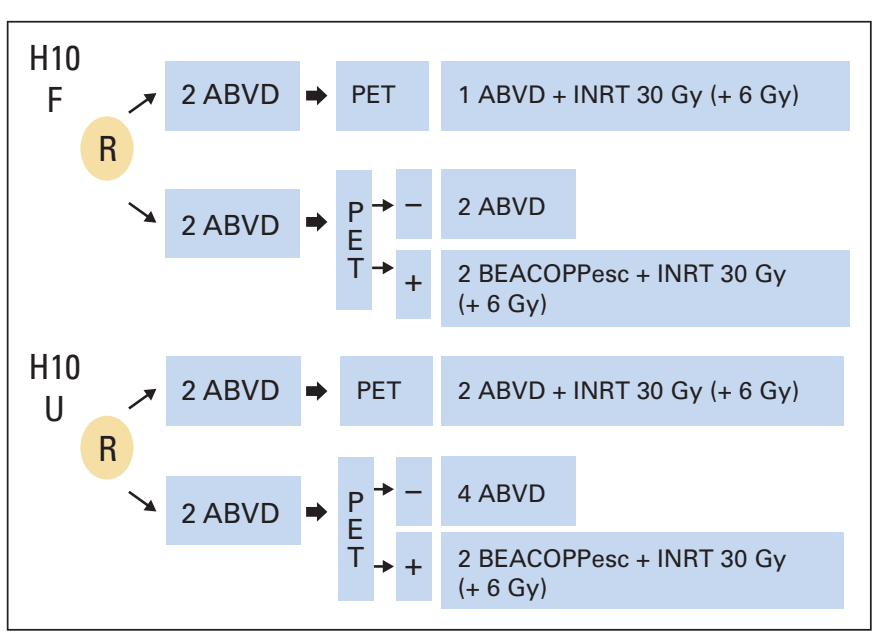

Fig 1. Study design of European Organisation for Research and Treatment of Cancer, Lymphoma Study Association, and Fondazione Italiana Linfomi H10 20551 trial of patients age 15 to 70 years with untreated supradiaphragmatic clinical stage I/II Hodgkin lymphoma (excluding nodular lymphocyte-predominant Hodgkin lymphoma). Favorable (F) status indicates age $<50$ years with $\leq$ three involved nodal areas, absence of mediastinal bulk (mediastinum-to-thorax ratio $<$ 0.35), and erythrocyte sedimentation rate (ESR) $<50 \mathrm{~mm}$ without B symptoms or ESR $<30 \mathrm{~mm}$ with B symptoms. Unfavorable (U) status indicates age $\geq 50$ years, $>$ four involved nodal areas, presence of mediastinal bulk (mediastinumto-thorax ratio $\geq 0.35$ ), or $E S R \geq 50 \mathrm{~mm}$ without $B$ symptoms or $E S R \geq 30 \mathrm{~mm}$ with B symptoms. ABVD, doxorubicin, bleomycin, vinblastine, and dacarbazine; BEACOPPesc, bleomycin, etoposide, doxorubicin, cyclophosphamide, vincristine, procarbazine, and prednisone escalated; INRT, involved-node radiotherapy; $\mathrm{PET}$, positron emission tomography; $\mathrm{R}$, random assignment.

reading, the local result of the early PET scan was decisional for further treatment in the experimental arm (first PET interpretation). A blinded second central PET review (second central review) was performed retrospectively after the recommendations of the independent data monitoring committee (IDMC) by four experts on the scans of 52 patients with events (including patients with early PET-negative and early PET-positive scans) and 52 randomly selected patients without events using a dedicated workstation (POSITOSCOPE; Keosys, Saint-Herblain, France). Twenty of these 104 scans could not be used for second central review for logistic or technical reasons; 84 were compared with the results of the first review (first central interpretation). Two experts from LYSA reviewed EORTC/FIL scans and vice versa. PET images were scored according to the International Harmonisation Project criteria, ${ }^{22}$ which were the generally accepted criteria for post-treatment assessment at the start of our trial. According to these criteria, a negative PET corresponds to scores 1 (no uptake) and 2 (uptake $\leq$ mediastinum) on the 5-point Deauville scale. ${ }^{23}$

\section{Statistics}

The primary end point was PFS, defined-from the date of random assignment to date of progression-as relapse after previous complete remission or progression after reaching partial remission $(\geq 50 \%$ decrease and resolution of B symptoms and no new lesions) or progressive disease (50\% increase from nadir of any previous partial remission lesions or appearance of new lesions) on computed tomography scan measurements during protocol treatment or death resulting from any cause, whichever occurred first (censoring rules are given in the Data Supplement).

The primary objective of the study was to evaluate whether the investigational treatment without RT was noninferior to the standard combinedmodality approach in terms of PFS. Each prognostic subgroup (favorable and unfavorable) was to be analyzed separately, using a log-rank test stratified by stage (I $v$ II) and availability of baseline PET, with a one-sided significance level of .025. Assuming a 5-year PFS in the standard arm of 95\% for the favorable subgroup and $90 \%$ for the unfavorable subgroup, and allowing for a decrease of $10 \%$ (to $85 \%$ and $80 \%$, respectively), the resulting noninferiority margins 


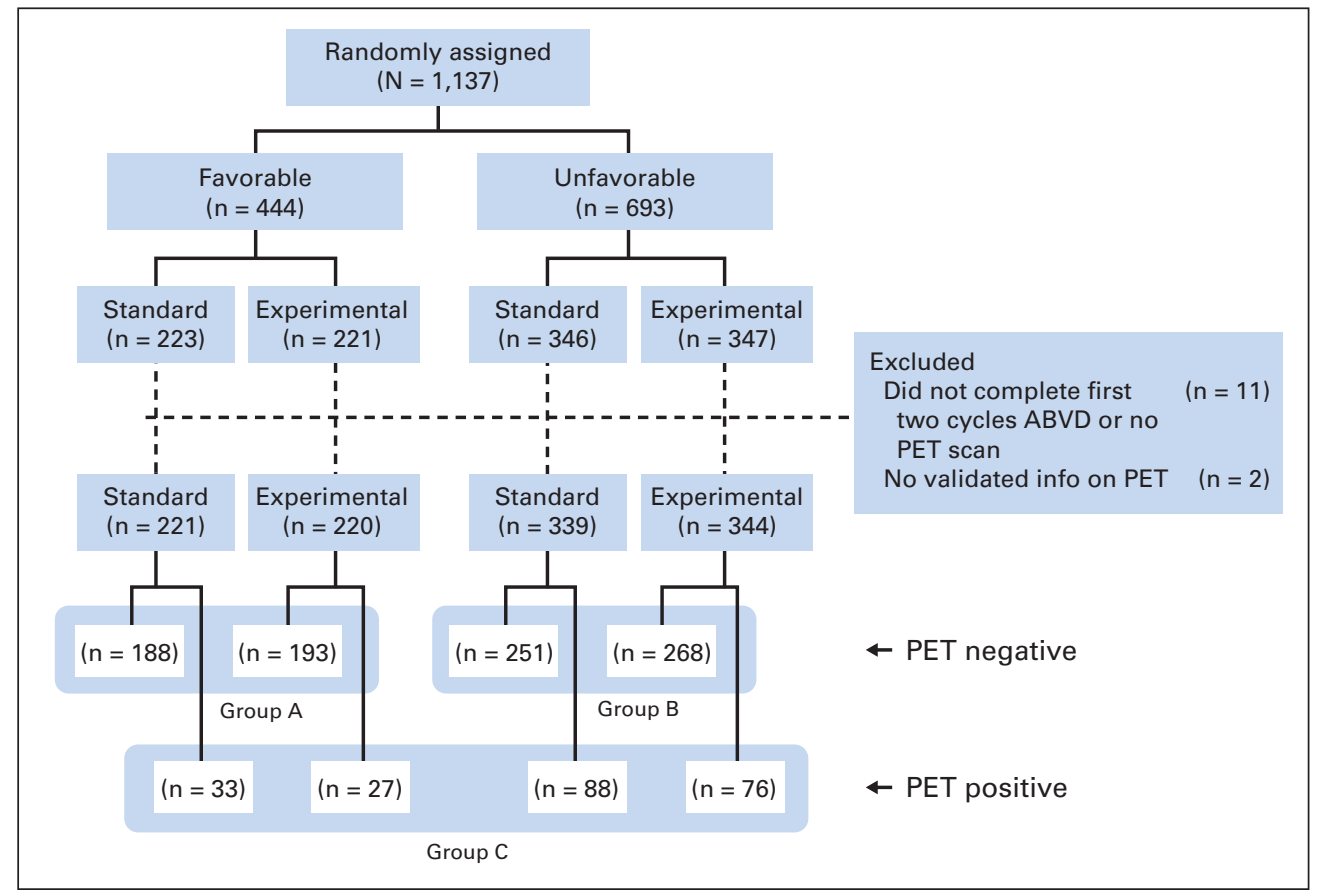

Fig 2. Flowchart of patients included in interim analysis. Group A, favorable early positron emission tomography (PET) negative; group $B$, unfavorable early $P E T$ negative; group $\mathrm{C}$, early PET positive (including both favorable and unfavorable). ABVD, doxorubicin, bleomycin, vinblastine, and dacarbazine.

for the hazard ratios (HRs) were 3.2 and 2.1 for favorable and unfavorable subgroups, respectively. A total of 26 and 63 events (progressions or deaths) for favorable and unfavorable subgroups, respectively, were required for $80 \%$ power to reject the null hypothesis of inferiority if the true HR were equal to 1.0. Sample size computations allowed for one interim analysis for futility. For the whole trial, we had planned to recruit 608 and 720 patients, respectively, in 4.5 years. The final analysis was scheduled to be performed after an additional follow-up of 2 years. No adjustment for multiplicity was made, because the trial is considered as two independent studies (favorable/unfavorable) embedded in the same protocol.

An interim futility analysis ${ }^{24-26}$ of the primary end point was scheduled after documentation of 12 and 22 events (progression, relapse, or death) for early PET-negative favorable and unfavorable subgroups, respectively. It was estimated that at that time point, roughly two thirds of the total number of required patients would be accrued. The purpose of the interim futility analysis was to stop the recruitment of favorable and unfavorable patients in case of inferiority of the investigational arm. Because testing for futility in this noninferiority trial corresponds to testing the hypothesis of no difference between the two arms, a one-sided superiority test was conducted, with a significance level determined by a Lan-DeMets spending function (Pocock like) based on the total error of 0.20 (details are given in the Data Supplement). Stopping boundaries were computed using East software (version 5.3; Cytel, Cambridge, MA). Statistical analysis of the study data was conducted using SAS software (version 9.1; SAS Institute, Cary, NC). The results of the early PETpositive group of patients lie outside the scope of this article.

\section{RESULTS}

The database was locked for the interim analysis at April 19,2010. The analysis was based on patients randomly assigned on or before August 31,2009 , at which time a total of 1,137 patients had been randomly assigned, with $61 \%, 25 \%$, and $14 \%$ recruited by LYSA, EORTC, and FIL respectively. Median follow-up at the clinical cutoff date (October $31,2009)$ was 1.1 years.
A baseline PET scan was performed in $93.2 \%$ of patients. Figure 2 shows the flowchart for patients enrolled in the interim analysis (favorable subgroup, $\mathrm{n}=444$; unfavorable subgroup, $\mathrm{n}=693$ ). In the favorable subgroup, $85.8 \%$ ( 381 of 444 ) had a negative early PET scan, and in the unfavorable subgroup, $74.8 \%$ (519 of 693) had one. The frequency of early PET-negative scans was somewhat higher in the experimental arm $(81.2 \%)$ than in the standard arm (77.2\%). Clinical characteristics and durations of follow-up were well balanced between the standard and experimental arms (Table 1). The interim futility analysis was performed among 1,124 of the 1,137 patients. Eleven patients did not complete the first two cycles of ABVD or had no early PET scan performed, and for two patients, no validated data were available.

\section{Favorable Early PET-Negative Group}

In the standard arm, 188 patients were included; in the experimental group, 193 patients (Table 2). A total of 10 events occurred (standard arm, one event $v$ experimental arm, nine events). All events were disease progressions (no deaths). On the basis of the actual information fraction (10 of 26 events required for final analysis) and the resulting one-sided significance level to perform the statistical test (.102), futility was declared $(P=.017[$ ie, $<.102])$; that is, on the basis of this analysis, it was unlikely that we could show equivalent efficacy between the standard and experimental treatments when continuing accrual to the originally planned total numbers. The estimated HR was 9.36 (79.6\% CI, 2.45 to 35.73$)$. PFS rates at 1 year were $100.0 \%$ and $94.9 \%$ in the standard and experimental arms, respectively.

\section{Unfavorable Early PET-Negative Group}

In the standard arm, 251 patients were included; in the experimental group, 268 patients (Table 2). A total of 23 events occurred (standard arm, seven events $v$ experimental arm, 16 


\begin{tabular}{|c|c|c|c|c|c|c|c|c|c|c|}
\hline \multirow[b]{3}{*}{ Characteristic } & \multicolumn{4}{|c|}{ Favorable } & \multicolumn{4}{|c|}{ Unfavorable } & \multirow{2}{*}{\multicolumn{2}{|c|}{ Total $(\mathrm{N}=900)$}} \\
\hline & \multicolumn{2}{|c|}{$\begin{array}{l}\text { Standard } \\
(n=188)\end{array}$} & \multicolumn{2}{|c|}{$\begin{array}{l}\text { Experimental } \\
(n=193)\end{array}$} & \multicolumn{2}{|c|}{$\begin{array}{l}\text { Standard } \\
(n=251)\end{array}$} & \multicolumn{2}{|c|}{$\begin{array}{l}\text { Experimental } \\
(n=268)\end{array}$} & & \\
\hline & No. & $\%$ & No. & $\%$ & No. & $\%$ & No. & $\%$ & No. & $\%$ \\
\hline \multicolumn{11}{|l|}{ Age, years } \\
\hline Median & \multirow{2}{*}{\multicolumn{2}{|c|}{$\begin{array}{c}29.5 \\
16-49\end{array}$}} & \multirow{2}{*}{\multicolumn{2}{|c|}{$\begin{array}{c}31 \\
15-49\end{array}$}} & \multicolumn{2}{|c|}{33} & \multirow{2}{*}{\multicolumn{2}{|c|}{$\begin{array}{c}31 \\
16-70\end{array}$}} & \multicolumn{2}{|c|}{31} \\
\hline Range & & & & & & & & & & \\
\hline Male sex & 107 & 56.9 & 97 & 50.3 & 124 & 49.4 & 123 & 45.9 & 451 & 50.1 \\
\hline Ann Arbor clinical stage I & 44 & 23.4 & 43 & 22.3 & 28 & 11.2 & 35 & 13.1 & 150 & 16.7 \\
\hline B symptoms & 13 & 6.9 & 13 & 6.7 & 90 & 35.9 & 111 & 41.4 & 227 & 25.2 \\
\hline \multicolumn{11}{|l|}{ No. of nodal areas } \\
\hline Median & \multicolumn{2}{|c|}{2} & \multicolumn{2}{|c|}{2} & \multicolumn{2}{|c|}{3} & \multicolumn{2}{|c|}{3} & \multicolumn{2}{|c|}{2} \\
\hline Range & \multicolumn{2}{|c|}{$1-3$} & \multicolumn{2}{|c|}{$1-3$} & \multicolumn{2}{|c|}{$1-5$} & \multicolumn{2}{|c|}{$1-5$} & \multicolumn{2}{|c|}{$1-5$} \\
\hline Bulky mediastinum & 0 & 0.0 & 0 & 0.0 & 101 & 40.2 & 106 & 39.5 & 207 & 23.0 \\
\hline \multicolumn{11}{|l|}{ MT ratio } \\
\hline Median & \multicolumn{2}{|c|}{0.3} & \multicolumn{2}{|c|}{0.3} & & & & & & \\
\hline Range & & & & & & & & & & \\
\hline $\mathrm{ESR}, \mathrm{mm} / \mathrm{hr}$ & & & & & & & & & & \\
\hline Overall & & & & & & & & & & \\
\hline Median & & & & & & & & & & \\
\hline Range & & & & & & & & & & \\
\hline No B symptoms & & & & & & & & & & \\
\hline Median & & & & & & & & & & \\
\hline Range & & & & & & & & & & \\
\hline B symptoms & & & & & & & & & & \\
\hline Median & & & & & & & & & & \\
\hline Range & & & & & & & & & & \\
\hline Baseline PET & 176 & 93.6 & 180 & 93.3 & 231 & 92.0 & 250 & 93.3 & 837 & 93.0 \\
\hline WHO PS 1-2 & 16 & 8.5 & 9 & 4.7 & 43 & 17.1 & 58 & 21.6 & 126 & 14.0 \\
\hline Duration of follow-up, yea & & & & & & & & & & \\
\hline Median & & & & & & & & & & \\
\hline Range & & & & & & & & & & \\
\hline
\end{tabular}

events). One patient died as a result of toxicity without signs of progression; all remaining events were progressions. On the basis of the actual information fraction ( 23 of 63 events required for final analysis) and the resulting one-sided significance level to perform the statistical test (.098), futility was declared $(P=$ $.026[\mathrm{ie},<.098])$. The estimated HR was $2.42(80.4 \% \mathrm{CI}, 1.35$ to 4.36). PFS rates at 1 year were $97.3 \%$ and $94.7 \%$ in the standard and experimental arms, respectively.

\section{Conclusion of IDMC and Recommendations}

Because the interim futility analysis showed statistical significance for PFS in both favorable and unfavorable early PET-negative groups, the IDMC recommended closing the study for continued accrual in the early PET-negative experimental arm. Moreover, it recommended changing treatment in patients with early PETnegative scans who were randomly assigned to the experimental arm and had not yet completed treatment from chemotherapy

Table 2. Results of Interim Analysis in Patients With Early PET-Negative Disease

\begin{tabular}{|c|c|c|c|c|c|c|c|}
\hline \multirow[b]{2}{*}{ Subset } & \multirow[b]{2}{*}{ No. of Patients } & \multirow[b]{2}{*}{ No. of Observed Events } & \multirow[b]{2}{*}{$\mathrm{HR}$} & \multirow[b]{2}{*}{ Adjusted $\mathrm{Cl}^{*}$} & \multirow[b]{2}{*}{$P+$} & \multicolumn{2}{|c|}{ 1-Year PFS } \\
\hline & & & & & & $\%$ & Adjusted $\mathrm{Cl}^{*}$ \\
\hline Favorable & & & & & .017 & & \\
\hline Standard & 188 & 1 & 1.00 & & & 100.00 & \\
\hline Experimental & 193 & 9 & 9.36 & 2.45 to 35.73 & & 94.93 & 91.89 to 96.85 \\
\hline Unfavorable & & & & & .026 & & \\
\hline Standard & 251 & 7 & 1.00 & & & 97.28 & 95.17 to 98.48 \\
\hline Experimental & 268 & 16 & 2.42 & 1.35 to 4.36 & & 94.70 & 92.11 to 96.46 \\
\hline
\end{tabular}


alone to the standard combined-modality approach, if possible. The interim analysis of early PET-positive patients did not meet the criteria for conclusion on the superiority of treatment intensification. Hence, the second question of the trial has not yet been answered at this interim analysis.

Because not all patients underwent a prospective first central review, and because of the somewhat higher frequency of early PET negativity in the experimental arm as compared with the standard arm, the IDMC recommended performing a blinded second central review before results of the interim analysis could be reported. This second central review was performed on early PET scans of all patients experiencing an event and, as a control group, randomly selected early PET scans of patients who did not experience an event.

\section{Actions Taken on IDMC Recommendations}

The study investigators were informed by letter on the main conclusion of the interim analysis (ie, stopping accrual in early PETnegative part of trial). It was advised that all patients who had already been randomly assigned to the experimental arm but who had not yet completed treatment or who had completed chemotherapy within the last 6 weeks be switched to standard-arm treatment (ie, chemotherapy plus IN-RT). New patients could still be enrolled and randomly assigned in the trial, but only for the early PET-positive part of the study. Therefore, patients with an early PET-negative scan after two cycles of ABVD would receive the combined-modality treatment, even if they had been assigned to the experimental arm at the start of the trial. Only patients with an early PET-positive scan remained in their assigned arm.

To ensure sufficient statistical power to address the early PETpositive objective of the trial, we decided to increase the number of required patients for the early PET-positive group from 248 to 335 patients. This revision was recommended by a senior statistician independent from the study team who had access to the actual recruitment rate and observed event rate in the standard arm. The study was closed to recruitment in June 2011, with a total of 1,952 randomly assigned patients.

\section{Second Central Review of Early PET Scans}

Overall, 70\% of patients were early PET negative at the first PET interpretation. At the second central review, six patients included in the interim analysis of early PET-negative patients would have changed to early PET positive. Excluding these patients from the interim analysis, numbers of events in the favorable group changed from one versus nine to one versus seven; in the unfavorable group, numbers change from seven versus 16 to five versus 14. The IDMC concluded that considering these changes, the advantage for the standard arm persisted.

\section{DISCUSSION}

The prescheduled interim futility analysis of the first randomized trial, to our knowledge, on early PET scan-driven treatment adaptation in clinical stage I/II HL revealed that more early progressions occurred after chemotherapy alone than after standard chemotherapy plus IN-RT in early PET-negative patients. This conclusion held for both the favorable and unfavorable subsets of patients. Although the numbers of events were small, and the median follow-up time was short, the coordinating study team decided, in line with the conclusions of the IDMC, to stop enrollment in the early PET-negative part of the study and to maintain combined-modality treatment as standard for patients with early PET-negative scans. The results of the blinded second central review of early PET scans did not change these conclusions. Importantly, the overall early outcome, both in the standard and experimental arms, was excellent.

The objective of our $\mathrm{H} 10$ trial was to determine a robust clinical tool for selecting patients who could be spared the toxicity of RT without compromising tumor control. On the basis of the futility interim analysis, it was unlikely that the study would demonstrate noninferiority for chemotherapy alone in early PET-negative patients. Thus, omitting IN-RT in early PET-negative patients resulted in a higher percentage of early relapses than combined-modality treatment.

The interim futility analysis was designed to stop recruitment if there were significant signals of inferiority of the investigational arm as compared with the standard arm. Although this was the conclusion, some questions can be raised. Whether the interim analysis was premature, in the sense that follow-up time (median, 1.1 years) was short and the number of events small, can be argued. The noninferiority margin was based on the assumption that this difference in progression would outweigh the burden of late toxicity. Admittedly, both arms had excellent early outcomes, and large proportions of patients did well with ABVD alone. However, the main goal of our trial was to find the ideal tool for early detection of patients who do not need RT; in this setting, early PET scanning did not meet our objective. Another matter of debate is whether the choice of statistical criteria for declaring futility was sound. However, given the interim data, there was a low likelihood that the experimental treatment arm would be found to be noninferior to the standard arm had the trial continued. Given the accumulated data, and assuming the observed HR trend would be maintained during the remainder of the trial, the conditional power to demonstrate noninferiority at the final analysis was $0 \%$ in both favorable and unfavorable groups. In the rather optimistic scenario where HR would be equal to 1.0 during the remainder of the trial, the conditional power to demonstrate noninferiority would be $3 \%$ and $30 \%$ in favorable and unfavorable subgroups, respectively.

Several randomized studies comparing chemotherapy alone with combined-modality treatment without using early PET scans have reached comparable conclusions. The EORTC/GELA H9 study on patients with favorable clinical stage I/II disease showed a significantly higher relapse rate in patients receiving chemotherapy alone as compared with those who received 20- or 36-Gy involved-field RT. ${ }^{7}$ The Canadian-US Intergroup study, with a somewhat different riskadapted design, also revealed higher risk of relapse in patients who did not undergo irradiation, ${ }^{5}$ although there were higher toxicity and death rates in the RT arm. It should be emphasized that subtotal nodal RT was used in this trial, an RT-strategy that has since been replaced by the less extensive IF-RT and, more recently, by IN-RT, as used in our H10 study. In a recent meta-analysis on combined-modality treatment versus chemotherapy alone, the combined approach resulted in improved tumor control as well as better overall survival. ${ }^{27} \mathrm{~A}$ survival advantage for combined-modality treatment compared with chemotherapy alone was demonstrated in a recent large populationbased study. ${ }^{28}$

A large proportion of patients with favorable clinical stage I/II disease can be treated with chemotherapy alone, as demonstrated in this trial. Patients who relapse after chemotherapy alone can often, 
although not always, benefit from intensive salvage treatment. It remains difficult, if not impossible, to balance the advantages of immediate cure with increased toxicity against a higher relapse risk after less intensive first-line treatment with its consequences for intensive salvage therapy. Thus, the correct treatment strategy is in the eye of the beholder. The recently completed UK RAPID LH2007 trial ${ }^{29}$ and the ongoing German Hodgkin Study Group HD16 and HD17 randomized trials, will contribute to the positioning of early PET scanning. Our interim analysis showed that the risk of early relapse in patients not undergoing irradiation was significantly higher than that after combined-modality treatment in early PET-negative patients, even though both treatment approaches resulted in excellent early outcome. Final trial analysis will reveal mature outcome data.

\section{AUTHORS' DISCLOSURES OF POTENTIAL CONFLICTS} OF INTEREST

The author(s) indicated no potential conflicts of interest.

\section{AUTHOR CONTRIBUTIONS}

Conception and design: John M.M. Raemaekers, Marc P.E. André, Massimo Federico, Theodore Girinsky, Pauline Brice, Christophe Fermé,
Richard van der Maazen, Reda Bouabdallah, Catherine J. Sebban, Yolande Lievens, Pieternella J. Lugtenburg, Rene-Olivier Casasnovas, Gustaaf van Imhoff, Tiana Raveloarivahy, Annibale Versari,

Catherine Fortpied

Administrative support: John M.M. Raemaekers, Michel Meignan, Catherine Fortpied

Provision of study materials or patients: John M.M. Raemaekers, Massimo Federico, Theodore Girinsky, Reman Oumedaly, Ercole Brusamolino, Pauline Brice, Richard van der Maazen, Manuel Gotti, Reda Bouabdallah, Aspasia Stamatoullas, Frank Morschhauser,

Pieternella J. Lugtenburg, Rene-Olivier Casasnovas, Gustaaf van Imhoff, Thierry van der Borght, Stephane Bardet, Annibale Versari

Collection and assembly of data: John M.M. Raemaekers, Marc P.E. André, Massimo Federico, Theodore Girinsky, Reman Oumedaly, Ercole Brusamolino, Manuel Gotti, Alessandro Re, Aspasia Stamatoullas, Frank Morschhauser, Elisabetta Abruzzese, Pierre Olivier, Gustaaf van Imhoff, Tiana Raveloarivahy, Thierry van der Borght, Stephane Bardet, Michel Meignan, Catherine Fortpied

Data analysis and interpretation: John M.M. Raemaekers, Marc P.E. André, Massimo Federico, Theodore Girinsky, Ercole Brusamolino, Richard van der Maazen, Tiana Raveloarivahy, Monica Bellei, Thierry van der Borght, Stephane Bardet, Annibale Versari, Martin Hutchings, Catherine Fortpied

Manuscript writing: All authors

Final approval of manuscript: All authors

\section{REFERENCES}

1. Fermé $\mathrm{C}$, Eghbali $\mathrm{H}$, Meerwaldt $\mathrm{JH}$, et al: Chemotherapy plus involved-field radiation in earlystage Hodgkin's disease. N Engl J Med 357:19161927, 2007

2. Engert $A$, Plütschow $A$, Eich $H T$, et al: Reduced treatment intensity in patients with early-stage Hodgkin's lymphoma. N Engl J Med 363:640-652, 2010

3. Girinsky $T$, van der Maazen R, Specht $L$, et al: Involved-node radiotherapy (INRT) in patients with early Hodgkin lymphoma: Concepts and guidelines. Radiother Oncol 79:270-277, 2006

4. Aleman BM, van den Belt-Dusebout AW, Klokman WJ, et al: Long-term cause-specific mortality of patients treated for Hodgkin's disease. $\mathrm{J}$ Clin Oncol 21:3431-3439, 2003

5. Meyer RM, Gospodarowicz MK, Connors JM, et al: ABVD alone versus radiation-based therapy in limited-stage Hodgkin's lymphoma. N Engl J Med 366:399-408, 2012

6. Herbst C, Rehan FA, Skoetz N, et al: Chemotherapy alone versus chemotherapy plus radiotherapy for early stage Hodgkin lymphoma. Cochrane Database Syst Rev 2:CD007110, 2011

7. Thomas J, Fermé C, Noordijk E, et al: Results of the EORTC-GELA H9 randomized trials: The H9-F trial (comparing 3 radiation dose levels) and $\mathrm{Hg}-\mathrm{U}$ trial (comparing 3 chemotherapy schemes) in patients with favorable or unfavorable early stage Hodgkin's lymphoma. Haematologica 92, 2007 (abstr C010)

8. Yahalom J: Don't throw out the baby with the bathwater: On optimizing cure and reducing toxicity in Hodgkin's lymphoma. J Clin Oncol 24:544-548, 2006

9. van der Maazen RW, Raemaekers JM: Chemotherapy and radiotherapy in Hodgkin's lymphoma: Joining in or splitting up? Curr Opin Oncol 18:660-666, 2006

10. Straus DJ: Chemotherapy only for localized Hodgkin lymphoma. J Intern Med 270:197-205, 2011
11. Younes A: Early-stage Hodgkin's lymphoma: In pursuit of perfection. J Clin Oncol 30:895-896, 2012

12. Brusamolino $E$, Baio A, Orlandi $E$, et al: Longterm events in adult patients with clinical stage IA-IIA nonbulky Hodgkin's lymphoma treated with four cycles of doxorubicin, bleomycin, vinblastine, and dacarbazine and adjuvant radiotherapy: A singleinstitution 15-year follow-up. Clin Cancer Res 12: 6487-6493, 2006

13. Hutchings $M$, Mikhaeel NG, Fields PA, et al: Prognostic value of interim FDG-PET after two or three cycles of chemotherapy in Hodgkin lymphoma. Ann Oncol 16:1160-1168, 2005

14. Gallamini A, Rigacci $L$, Merli F, et al: The predictive value of positron emission tomography scanning performed after two courses of standard therapy on treatment outcome in advanced stage Hodgkin's disease. Haematologica 91:475-481, 2006

15. Hutchings $M$, Loft $A$, Hansen $M$, et al: FDGPET after two cycles of chemotherapy predicts treatment failure and progression-free survival in Hodgkin lymphoma. Blood 107:52-59, 2006

16. von Tresckow $B$, Plütschow $A$, Fuchs $M$, et al: Dose-intensification in early unfavorable Hodgkin's lymphoma: Final analysis of the German Hodgkin Study Group HD14 trial. J Clin Oncol 30:907-913, 2012

17. André $M$, Reman $O$, Federico $M$, et al: First report on the H10 EORTC/GELA/IIL randomized intergroup trial on early FDG-PET scan guided treatment adaptation versus standard combined modality treatment in patients with supradiaphragmatic stage I/II Hodgkin's lymphoma, for the Groupe d'Etude des Lymphomes de I'Adulte (GELA), European Organisation for the Research and Treatment of Cancer (EORTC) Lymphoma Group and the Intergruppo Italiano Linfomi (IIL). Blood 114, 2009 (abstr 97)

18. Tubiana $M$, Henry-Amar $M$, van der WerfMessing B, et al: A multivariate analysis of prognostic factors in early stage Hodgkin's disease. Int J Radiat Oncol Biol Phys 11:23-30, 1985
19. Noordijk EM, Carde $P$, Dupouy $N$, et al: Combined-modality therapy for clinical stage I or II Hodgkin's lymphoma: Long-term results of the European Organisation for Research and Treatment of Cancer H7 randomized controlled trials. J Clin Oncol 24:3128-3135, 2006

20. European Organisation for Research and Treatment of Cancer, Lymphoma Study Association,

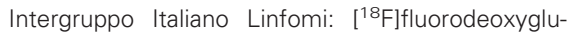
cose PET scan-guided therapy or standard therapy in treating patients with previously untreated stage I or stage II Hodgkin's lymphoma. http://clinicaltrials.gov/show/NCT00433433

21. Meignan $M$, Itti $E$, Bardet $S$, et al: Development and application of a real-time on-line blinded independent central review of interim PET scans to determine treatment allocation in lymphoma trials. $\mathrm{J}$ Clin Oncol 27:2739-2741, 2009

22. Juweid ME, Stroobants S, Hoekstra OS, et al: Use of positron emission tomography for response assessment of lymphoma: Consensus of the Imaging Subcommittee of International Harmonisation Project in Lymphoma. J Clin Oncol 25:571-578, 2007

23. Meignan $M$, Gallamini $A$, Haioun $C$, et al: Report on the Second International Workshop on Interim Positron Emission Tomography in Lymphoma held in Menton, France, 8-9 April 2010. Leuk Lymphoma 51:2171-2180, 2010

24. Demets DL: Futility approaches to interim monitoring by data monitoring committees. Clin Trials 3:522-529, 2006

25. Snapinn S, Chen MG, Jiang $Q$, et al: Assessment of futility in clinical trials. Pharm Stat 5:273281, 2006

26. Cairns JA, Wittes J, Wyse DG, et al: Monitoring the ACTIVE-W trial: Some issues in monitoring a noninferiority trial. Am Heart J 155:33-41, 2008

27. Herbst C, Rehan FA, Brillant $C$, et al: Combined modality treatment improves tumor control and overall survival in patients with early stage 
Hodgkin's lymphoma: A systematic review. Haematologica 95:494-500, 2010

28. Koshy M, Rich SE, Mahmood U, et al: Declining use of radiotherapy in stage I and II Hodgkin's disease and its effect on survival and secondary malignancies. Int J Radiat Oncol Biol Phys 82:619-625, 2012

29. Radford J, Barrington S, Counsell N, et al: Involved field radiotherapy versus no further treat- ment in patients with clinical stage IA and IIA Hodgkin lymphoma and a negative PET scan after 3 cycles of ABVD: Results of the UK NCRI RAPID trial. Blood 120, 2012 (abstr 547)

\section{Affiliations}

John M.M. Raemaekers and Richard van der Maazen, Radboud University Medical Center, Nijmegen; Pieternella J. Lugtenburg, Erasmus University Medical Center, Rotterdam; Gustaaf van Imhoff, University Medical Centre Groningen, Groningen, the Netherlands; Marc P.E. André and Thierry van der Borght, Centre Hospitalier Universitaire (CHU) L'Université Catholique de Louvain Mont Godinne, Yvoir; Yolande Lievens, Ghent University Hospital, Ghent; Tiana Raveloarivahy and Catherine Fortpied, European Organisation for Research and Treatment of Cancer, Brussels, Belgium; Massimo Federico and Monica Bellei, University of Modena and Reggio Emilia, Modena; Ercole Brusamolino, Istituto Clinico Humanitas, Istituto di Ricovero e Cura a Carattere Scientifico (IRCCS), Milan; Manuel Gotti, Fondazione IRCCS Policlinico San Matteo, Pavia; Allessandro Re, Spedali Civili Hospital, Brescia; Elisabetta Abruzzese, San Eugenio Hospital, Tor Vergata University, Rome; Annibale Versari, Arcispedale Santa Maria Nuova, IRCCS di Reggio Emilia, Reggio Emilia, Italy; Theodore Girinsky and Christophe Fermé, Institut Gustave Roussy, Villejuif; Reman Oumedaly, CHU Caen; Stephane Bardet, Centre Francois Baclesse, Caen; Pauline Brice, Hôpital Saint-Louis, Paris; Reda Bouabdallah, Institut Paoli Calmette, Marseille; Catherine J. Sebban, Centre Léon Bérard, Lyon; Aspasia Stamatoullas, Centre Henri Becquerel, Rouen; Frank Morschhauser, CHU de Lille, Lille; Pierre Olivier, CHU Nancy, Nancy; Rene-Olivier Casasnovas, CHU de Dijon, Dijon; Michel Meignan, Hôpital Henri Mondor, Creteil, France; and Martin Hutchings, Rigshospitalet, Kopenhagen, Denmark.

\section{ASCO Annual Meeting}

Join us for the 2014 ASCO Annual Meeting (May 30-June 3 in Chicago, IL), which brings together more than 25,000 oncology professionals from a wide range of specialties. Marking the 50th Anniversary of ASCO, this year's theme is Science and Society... The Next 50 Years. As we embark on our next 50 years as an organization, we will reflect on the tremendous progress ASCO has made against cancer and look at the future of science in oncology. For more information, visit am.asco.org.

Each year, ASCO, in conjunction with our cosponsors, organizes a wide array of high-quality meetings, providing educational and scientific programs to advance your understanding of cancer. Join us for one or more of ASCO's meetings to interact with oncology experts, network with colleagues, and earn CME credit. 


\section{Acknowledgment}

We thank all investigators of the participating groups who actively entered patients in this trial, data managers, supporting crew, and, last but not least, all patients who participated. 